\title{
Remarks on Remnants by Fermions' Tunnelling from Black Strings
}

\author{
Deyou Chen and Zhonghua Li \\ Institute of Theoretical Physics, China West Normal University, Nanchong 637009, China \\ Correspondence should be addressed to Deyou Chen; dyouchen@gmail.com
}

Received 24 November 2013; Revised 19 January 2014; Accepted 19 January 2014; Published 13 March 2014

Academic Editor: Elias C. Vagenas

Copyright ( 2014 D. Chen and Z. Li. This is an open access article distributed under the Creative Commons Attribution License, which permits unrestricted use, distribution, and reproduction in any medium, provided the original work is properly cited. The publication of this article was funded by SCOAP S $^{3}$

\begin{abstract}
Hawking's calculation is unable to predict the final stage of the black hole evaporation. When effects of quantum gravity are taken into account, there is a minimal observable length. In this paper, we investigate fermions' tunnelling from the charged and rotating black strings. With the influence of the generalized uncertainty principle, the Hawking temperatures are not only determined by the rings, but also affected by the quantum numbers of the emitted fermions. Quantum gravity corrections slow down the increases of the temperatures, which naturally leads to remnants left in the evaporation.
\end{abstract}

\section{Introduction}

Hawking radiation is a quantum tunnelling phenomenon of particles across black holes' horizons. To describe this phenomenon, the semiclassical tunnelling method, which relies on calculating the imaginary part of emission particle's action, was put forward [1]. Adopting the WKB approximation, one can get the relationship between the tunnelling rate and the action of the classically forbidden trajectory of the particle. Here we adopt the canonically invariant expression $[2-4]$

$$
\Gamma \propto \exp [-\operatorname{Im} \oint p d r]
$$

This canonically invariant relation was first derived in [5-7].

The null geodesic method and the Hamilton-Jacobi method are usual methods employed to derive the imaginary part [8-12]. In the null geodesic method [8], we should first perform the Painleve coordinate transformation on a metric and then use canonical momenta and Hamilton canonical equations to get the imaginary part. When the variable background spacetime is taken into account, the corrected Hawking temperature is higher than the standard one. Therefore, the variable background spacetime implies the accelerated evaporation. The equation of motion of a massive particle is different from that of the massless one. The former obeys de Broglie wave function relation. Therefore, the phase velocity of the particle was adopted to research the tunnelling radiation of massive particles in the subsequent investigations $[13,14]$. The embryonic form of the HamiltonJacobi method [11] was first found in $[9,10]$. In this method, the action satisfies the Hamilton-Jacobi equation. Taking into account the property of the spacetime, one carries out separation of variables on the action. Then, inserting the separated variables into the Hamilton-Jacobi equation and solving it, one gets the imaginary part. Extending this work to the tunnelling radiation of fermions, the standard Hawking temperatures of the spherically symmetric and charged black holes were recovered [15]. Other works about fermions' tunnelling radiation are referred to in [16-24]. The standard temperatures were also recovered by anomaly cancellations [25-27].

Various theories of quantum gravity predict the existence of a minimal observable length [28-32]. This length can be implemented in the model of the generalized uncertainty principle (GUP)

$$
\Delta x \Delta p \geq \frac{\hbar}{2}\left[1+\beta \Delta p^{2}\right]
$$


where $\beta=\beta_{0}\left(l_{p}^{2} / \hbar^{2}\right), \beta_{0}$ is a dimensionless parameter, and $l_{p}$ is the Planck length. The derivation of the GUP relies on the modified fundamental commutation relations. Kempf et al. first modified commutation relations [33] and got $\left[x_{i}, p_{j}\right]=$ $i \hbar \delta_{i j}\left[1+\beta p^{2}\right]$, where $x_{i}$ and $p_{i}$ are operators of position and momentum defined by

$$
x_{i}=x_{0 i}, \quad p_{i}=p_{0 i}\left(1+\beta p^{2}\right),
$$

and $x_{0 i}$ and $p_{0 i}$ satisfy the canonical commutation relations $\left[x_{0 i}, p_{0 j}\right]=i \hbar \delta_{i j}$.

This modification plays an important role in quantum gravity. With considerations of modifications, the cosmological constant problem was discussed and the finiteness of the constant was derived in [34]. Using a new form of GUP, the Unruh effect has been analyzed in [35]. The quantum dynamics of the Friedmann-Robertson-Walker universe was gotten in [36]. The related predictions on postinflation preheating in the cosmology were derived in [37]. Using the modifications, the thermodynamics of the black holes were researched in [38-40] and the tunnelling radiation of scalar particles was investigated in [41]. In recent work [42], taking into account effects of quantum gravity, the authors modified the Dirac equation in curved spacetime and investigated fermions' tunnelling from the Schwarzschild black hole. They derived that the quantum correction slows down the increase of the Hawking temperature, which leads to the remnant.

In this paper, we extend this work to anti-de Sitter spacetime and investigate the tunnelling radiation of fermions from black strings, where effects of quantum gravity are taken into account. Black strings are cylindrically symmetric solutions of the Einstein-Maxwell equations with a negative cosmological constant. The solutions are asymptotically antide Sitter in transverse direction and along the axis. There are three Killing vectors, $\partial_{t}, \partial_{\theta}$, and $\partial_{z}$, as the minimal symmetry. The AdS/CFT correspondence is an important topic in modern physics. Researches of anti-de Sitter spacetime are helpful to understand this correspondence. To incorporate effects of quantum gravity, we first modify the Dirac equation in curved spacetime by operators of position and momentum defined in [33] and then adopt the HamiltonJacobi method to get the imaginary parts of the action. The corrected Hawking temperatures are not only determined by the mass, charge, and angular momentum of the strings, but also affected by the quantum numbers (charge, angular momentum, mass, and energy) of the emitted fermions. Quantum gravity corrections slow down the increases of the Hawking temperatures. It is natural to lead to the remnants left in the evaporation.

The rest is organized as follows. In the next section, using operators of position and momentum defined in [33], we modify the Dirac equation in curved spacetime. In Section 3, we investigate the tunnelling radiation of charged fermions from the charged black string. The remnant is observed in the evaporation. In Section 4, the radiation of uncharged fermions in the rotating black string is discussed. Section 5 is devoted to our conclusion.

\section{Generalized Dirac Equation}

Here we adopt the modified fundamental commutation relation put forward in [33] to modify the Dirac equation in curved spacetime. Using (3), the square of momentum operators is gotten as

$$
\begin{aligned}
p^{2} & =p_{i} p^{i}=-\hbar^{2}\left[1-\beta \hbar^{2}\left(\partial_{j} \partial^{j}\right)\right] \partial_{i} \cdot\left[1-\beta \hbar^{2}\left(\partial^{j} \partial_{j}\right)\right] \partial^{i} \\
& \simeq-\hbar^{2}\left[\partial_{i} \partial^{i}-2 \beta \hbar^{2}\left(\partial^{j} \partial_{j}\right)\left(\partial^{i} \partial_{i}\right)\right] .
\end{aligned}
$$

The higher order terms of $\beta$ are neglected in the last step. In the theory of quantum gravity, the generalized frequency is found as [43]

$$
\widetilde{\omega}=E\left(1-\beta E^{2}\right)
$$

where $E$ is the energy operator and is defined as $E=i \hbar \partial_{t}$. From the energy mass shell condition $p^{2}+m^{2}=E^{2}$, the generalized expression of the energy was derived [41, 43-45]. It is

$$
\widetilde{E}=E\left[1-\beta\left(p^{2}+m^{2}\right)\right] .
$$

The generalized Dirac equation without considerations of electromagnetic effects in the flat spacetime has been derived in [44] by the consequence of the GUP. In curved spacetime, the Dirac equation with an electromagnetic field takes on the form

$$
i \gamma^{\mu}\left(\partial_{\mu}+\Omega_{\mu}+\frac{i}{\hbar} e A_{\mu}\right) \Psi+\frac{m}{\hbar} \Psi=0,
$$

where $\Omega_{\mu} \equiv(i / 2) \omega_{\mu}{ }^{a b} \Sigma_{a b}, \omega_{\mu}{ }^{a b}$ is the spin connection defined by the tetrad $e^{\lambda}{ }_{b}$ and ordinary connection

$$
\omega_{\mu}^{a}{ }_{b}=e_{\nu}{ }^{a} e^{\lambda}{ }_{b} \Gamma_{\mu \lambda}^{\nu}-e_{b}^{\lambda} \partial_{\mu} e_{\lambda}{ }^{a} .
$$

The Latin indices live in the flat metric $\eta_{a b}$ while Greek indices are raised and lowered by the curved metric $g_{\mu \nu}$. The tetrad can be constructed from

$$
\begin{gathered}
g_{\mu \nu}=e_{\mu}{ }^{a} e_{\nu}{ }^{b} \eta_{a b}, \quad \eta_{a b}=g_{\mu \nu} e^{\mu}{ }_{a} e_{b}^{\nu}, \\
e^{\mu}{ }_{a} e_{\nu}{ }^{a}=\delta_{\nu}^{\mu}, \quad e^{\mu}{ }_{a} e_{\mu}{ }^{b}=\delta_{a}^{b} .
\end{gathered}
$$

In (7), $\Sigma_{a b}$ is the Lorentz spinor generators defined by

$$
\Sigma_{a b}=\frac{i}{4}\left[\gamma^{a}, \gamma^{b}\right], \quad\left\{\gamma^{a}, \gamma^{b}\right\}=2 \eta^{a b}
$$

Then one can construct $\gamma^{\mu}$ 's in the curved spacetime as

$$
\gamma^{\mu}=e^{\mu}{ }_{a}^{a} \gamma^{a}, \quad\left\{\gamma^{\mu}, \gamma^{\nu}\right\}=2 g^{\mu \nu} .
$$

To get the generalized Dirac equation in the curved spacetime, we rewrite (7) as

$$
-i \gamma^{0} \partial_{0} \Psi=\left(i \gamma^{i} \partial_{i}+i \gamma^{\mu} \Omega_{\mu}+i \gamma^{\mu} \frac{i}{\hbar} e A_{\mu}+\frac{m}{\hbar}\right) \Psi .
$$


Using (4), (6), and (12) and neglecting the higher order terms of $\beta$, we get $[41,43-45]$

$$
\begin{aligned}
-i \gamma^{0} \partial_{0} \Psi= & \left(i \gamma^{i} \partial_{i}+i \gamma^{\mu} \Omega_{\mu}+i \gamma^{\mu} \frac{i}{\hbar} e A_{\mu}+\frac{m}{\hbar}\right) \\
& \times\left(1+\beta \hbar^{2} \partial_{j} \partial^{j}-\beta m^{2}\right) \Psi,
\end{aligned}
$$

which is rewritten as

$$
\begin{aligned}
& {\left[i \gamma^{0} \partial_{0}+i \gamma^{i} \partial_{i}\left(1-\beta m^{2}\right)+i \gamma^{i} \beta \hbar^{2}\left(\partial_{j} \partial^{j}\right) \partial_{i}\right.} \\
& \quad+\frac{m}{\hbar}\left(1+\beta \hbar^{2} \partial_{j} \partial^{j}-\beta m^{2}\right) \\
& \quad+i \gamma^{\mu} \frac{i}{\hbar} e A_{\mu}\left(1+\beta \hbar^{2} \partial_{j} \partial^{j}-\beta m^{2}\right) \\
& \left.\quad+i \gamma^{\mu} \Omega_{\mu}\left(1+\beta \hbar^{2} \partial_{j} \partial^{j}-\beta m^{2}\right)\right] \Psi=0 .
\end{aligned}
$$

Thus the generalized Dirac equation is derived. When $A_{\mu}=$ 0 , it describes an equation without electromagnetic fields. In the following sections, we adopt (14) to describe fermions tunnelling from the charged and rotating black strings.

\section{Fermions' Tunnelling from a Charged Black String}

The 4-dimensional neutral black string solutions to EinsteinMaxwell equations with a negative cosmological constant were derived in [46]. Subsequently, the general solutions with electric charges were gotten [47]. In this section, we investigate charged fermions tunnelling from a cylindrically symmetric black string. The black string solution is given by [47]

$$
d s^{2}=-f(r) d t^{2}+\frac{1}{g(r)} d r^{2}+r^{2} d \theta^{2}+\alpha^{2} r^{2} d z^{2},
$$

with the electromagnetic potential

$$
A_{\mu}=\left(A_{t}, 0,0,0\right)=\left(\frac{2 Q}{\alpha r}, 0,0,0\right),
$$

where $f(r)=g(r)=\alpha^{2} r^{2}-(4 M / \alpha r)+\left(4 Q^{2} / \alpha^{2} r^{2}\right), 0 \leq$ $\theta \leq 2 \pi, \alpha^{2}=-\Lambda / 3$, and $\Lambda$ is the negative cosmology constant. $M$ and $Q$ are the ADM mass and charge per unit length in the $\mathrm{z}$ direction, respectively. The above spacetime is asymptotically anti-de Sitter in the transverse directions and string directions. The singularity at $r=0$ is enclosed by the horizon $r_{+}$if the condition $Q^{2} \leq(3 / 4) M^{4 / 3}$ holds. The event horizon $r_{+}$is located at

$$
r_{+}=\frac{1}{2}\left[\sqrt{2 R}+\left(-2 R+\frac{8 M}{\alpha^{3} \sqrt{2 R}}\right)\right],
$$

where

$$
\begin{aligned}
R= & {\left[\frac{M^{2}}{\alpha^{6}}+\left(\left(\frac{M^{2}}{\alpha^{6}}\right)^{2}-\left(\frac{4 Q^{2}}{3 \alpha^{4}}\right)^{3}\right)^{1 / 2}\right]^{1 / 3} } \\
& +\left[\frac{M^{2}}{\alpha^{6}}-\left(\left(\frac{M^{2}}{\alpha^{6}}\right)^{2}-\left(\frac{4 Q^{2}}{3 \alpha^{4}}\right)^{3}\right)^{1 / 2}\right]^{1 / 3} .
\end{aligned}
$$

The metric (15) describes a neutral black string solution when $Q=0$.

For a spin-1/2 fermion, there are two states corresponding to spin-up and spin-down. Here we only investigate the state with spin-up. The investigation of the state with spin-down is parallel and the same result can be obtained. To describe the motion of a charge fermion, we suppose that the wave function takes on the form

$$
\Psi=\left(\begin{array}{c}
A \\
0 \\
B \\
0
\end{array}\right) \exp \left(\frac{i}{\hbar} I(t, r, \theta, z)\right),
$$

where $A$ and $B$ are functions of $t, r, \theta$, and $z$ and $I$ is the action of the fermion with spin-up state. To find gamma matrices, we should first construct a tetrad. It is straightforward to construct a tetrad from the metric (15). The tetrad is

$$
e_{\mu}{ }^{a}=\operatorname{diag}(\sqrt{f}, 1 / \sqrt{g}, r, \alpha r) \text {. }
$$

Then gamma matrices are gotten as

$$
\begin{aligned}
\gamma^{t}=\frac{1}{\sqrt{f(r)}}\left(\begin{array}{cc}
i & 0 \\
0 & -i
\end{array}\right), & \gamma^{\theta}=\sqrt{g^{\theta \theta}}\left(\begin{array}{cc}
0 & \sigma^{1} \\
\sigma^{1} & 0
\end{array}\right), \\
\gamma^{r}=\sqrt{g(r)}\left(\begin{array}{cc}
0 & \sigma^{3} \\
\sigma^{3} & 0
\end{array}\right), & \gamma^{z}=\sqrt{g^{z z}}\left(\begin{array}{cc}
0 & \sigma^{2} \\
\sigma^{2} & 0
\end{array}\right) .
\end{aligned}
$$

In the above equations, $\sqrt{g^{\theta \theta}}=1 / r$ and $\sqrt{g^{z z}}=1 / \alpha r$. To apply the WKB approximation, we insert the wave function and the gamma matrices into the generalized Dirac equation and then divide by the exponential term and multiply by $\hbar$. The resulting equation of leading order in $\hbar$ is derived and decoupled into four equations

$$
\begin{aligned}
-i A & \frac{1}{\sqrt{f}} \partial_{t} I-B\left(1-\beta m^{2}\right) \sqrt{g} \partial_{r} I \\
& -A m \beta\left[g^{r r}\left(\partial_{r} I\right)^{2}+g^{\theta \theta}\left(\partial_{\theta} I\right)^{2}+g^{z z}\left(\partial_{z} I\right)^{2}\right] \\
& +B \beta \sqrt{g} \partial_{r} I\left[g^{r r}\left(\partial_{r} I\right)^{2}+g^{\theta \theta}\left(\partial_{\theta} I\right)^{2}+g^{z z}\left(\partial_{z} I\right)^{2}\right] \\
& +A m\left(1-\beta m^{2}\right)-i A \frac{e A_{t}}{\sqrt{f}} \\
& \times\left[1-\beta m^{2}-\left(g^{r r}\left(\partial_{r} I\right)^{2}+g^{\theta \theta}\left(\partial_{\theta} I\right)^{2}+g^{z z}\left(\partial_{z} I\right)^{2}\right)\right] \\
= & 0,
\end{aligned}
$$




$$
\begin{aligned}
& i B \frac{1}{\sqrt{f}} \partial_{t} I-A\left(1-\beta m^{2}\right) \sqrt{g} \partial_{r} I \\
&-B m \beta\left[g^{r r}\left(\partial_{r} I\right)^{2}+g^{\theta \theta}\left(\partial_{\theta} I\right)^{2}+g^{z z}\left(\partial_{z} I\right)^{2}\right] \\
&+A \beta \sqrt{g} \partial_{r} I\left[g^{r r}\left(\partial_{r} I\right)^{2}+g^{\theta \theta}\left(\partial_{\theta} I\right)^{2}+g^{z z}\left(\partial_{z} I\right)^{2}\right] \\
&+B m\left(1-\beta m^{2}\right)+i B \frac{e A_{t}}{\sqrt{f}} \\
& \quad \times\left[1-\beta m^{2}-\left(g^{r r}\left(\partial_{r} I\right)^{2}+g^{\theta \theta}\left(\partial_{\theta} I\right)^{2}+g^{z z}\left(\partial_{z} I\right)^{2}\right)\right] \\
&=0, \\
& A\left\{-\left(1-\beta m^{2}\right) \sqrt{g^{\theta \theta}} \partial_{\theta} I+\beta \sqrt{g^{\theta \theta}} \partial_{\theta} I\right. \\
& \times\left[g^{r r}\left(\partial_{r} I\right)^{2}+g^{\theta \theta}\left(\partial_{\theta} I\right)^{2}+g^{z z}\left(\partial_{z} I\right)^{2}\right] \\
&-i\left(1-\beta m^{2}\right) \sqrt{g^{z z}} \partial_{z} I+i \beta \sqrt{g^{z z}} \partial_{z} I \\
&\left.\quad \times\left[g^{r r}\left(\partial_{r} I\right)^{2}+g^{\theta \theta}\left(\partial_{\theta} I\right)^{2}+g^{z z}\left(\partial_{z} I\right)^{2}\right]\right\}=0 \\
& B\left\{-\left(1-\beta m^{2}\right) \sqrt{g^{\theta \theta}} \partial_{\theta} I+\beta \sqrt{g^{\theta \theta}} \partial_{\theta} I\right. \\
& \times {\left[g^{r r}\left(\partial_{r} I\right)^{2}+g^{\theta \theta}\left(\partial_{\theta} I\right)^{2}+g^{z z}\left(\partial_{z} I\right)^{2}\right] } \\
&- i\left(1-\beta m^{2}\right) \sqrt{g^{z z}} \partial_{z} I+i \beta \sqrt{g^{z z}} \partial_{z} I \\
&\left.\times\left[g^{r r}\left(\partial_{r} I\right)^{2}+g^{\theta \theta}\left(\partial_{\theta} I\right)^{2}+g^{z z}\left(\partial_{z} I\right)^{2}\right]\right\}=0
\end{aligned}
$$

Obviously, it is difficult to get the solution of the action $I$ from the above equations. However, the action can be separated by the property of the black string. Considering the Killing vectors of the spacetime, the author separated the action as $I=-\omega t+W(r)+l \theta+J z[17]$, where $\omega$ is the energy of the emitted fermion. From the above four equations, we carry out separation of variables as

$$
I=-\omega t+W(r)+\Theta(\theta, z) .
$$

We first observe (23) and find that they are irrelevant to $A$ and $B$ and can be reduced to the same equation. Inserting (24) into (23) yields

$$
\begin{aligned}
& \left(\sqrt{g^{\theta \theta}} \partial_{\theta} \Theta+i \sqrt{g^{z z}} \partial_{z} \Theta\right) \\
& \quad \times\left[1-\beta m^{2}-\beta g^{r r}\left(\partial_{r} W\right)^{2}-\beta g^{\theta \theta}\left(\partial_{\theta} \Theta\right)^{2}-\beta g^{z z}\left(\partial_{z} \Theta\right)^{2}\right] \\
& =0 .
\end{aligned}
$$

In the above equation, the summation of factors in the square brackets cannot be zero. Therefore, it should be

$$
\sqrt{g^{\theta \theta}} \partial_{\theta} \Theta+i \sqrt{g^{z z}} \partial_{z} \Theta=0
$$

which yields a complex function solution (other than the trivial constant solution) of $\Theta$. However, this solution has no contribution to the tunnelling rate. Therefore, we will not consider its contribution in the calculation. Another important relation predicted by $(26)$ is $g^{\theta \theta}\left(\partial_{\theta} \Theta\right)^{2}+g^{z z}\left(\partial_{z} \Theta\right)^{2}=$ 0 . Now we focus our attention on the first two equations. Inserting (24) into (22) and canceling $A$ and $B$ yield

$$
A_{6}\left(\partial_{r} W\right)^{6}+A_{4}\left(\partial_{r} W\right)^{4}+A_{2}\left(\partial_{r} W\right)^{2}+A_{0}=0,
$$

where

$$
\begin{aligned}
A_{6}= & \beta^{2} g^{3} f \\
A_{4}= & \beta g^{2} f\left(m^{2} \beta-2\right)-\beta^{2} g^{2} e^{2} A_{t}^{2}, \\
A_{2}= & g f\left(1-\beta m^{2}\right)\left(1+\beta m^{2}\right) \\
& -2 \beta g e A_{t}\left[\omega-e A_{t}\left(1-\beta m^{2}\right)\right], \\
A_{0}= & -m^{2} f\left(1-\beta m^{2}\right)^{2}-\left[\omega-e A_{t}\left(1-\beta m^{2}\right)\right]^{2} .
\end{aligned}
$$

Neglect the higher order terms of $\beta$ and solve (27) at the event horizon. Thus the imaginary part of the radial action is

$$
\begin{aligned}
I m W_{ \pm}= \pm \int & \frac{d r}{\sqrt{g f}} \sqrt{\left[\omega-e A_{t}\left(1-\beta m^{2}\right)\right]^{2}+m^{2} f} \\
& \times\left(1+\beta m^{2}+\beta \frac{\widetilde{\omega}_{0}^{2}-e A_{t} \widetilde{\omega}_{0}}{f}\right) \\
= \pm \pi & \frac{\omega-e A_{t+}}{f^{\prime}}(1+\beta \xi),
\end{aligned}
$$

where $+(-)$ denote the outgoing (ingoing) solutions, $f^{\prime}=$ $2 \alpha^{2} r_{+}+\left(4 M / \alpha r_{+}^{2}\right)-\left(8 Q^{2} / \alpha^{2} r_{+}^{3}\right), \xi=(3 / 2) m^{2}+\left(\left(2 m^{2}+\right.\right.$ $\left.1) / 2 \omega_{0}\right)+\left(2 e A_{t+} / f^{\prime} r_{+}\right)-(2 / 3)\left(\omega_{0} / f^{\prime} r_{+}\right), \widetilde{\omega}_{0}=\omega-e A_{t}$, $\omega_{0}=\omega-e A_{t+}$, and $A_{t+}=2 Q / \alpha r_{+}$is the electromagnetic potential at the event horizon. Using the relation of the roots of $f=0$, it is easily proved that $\xi>0$. The tunnelling rate of the charged fermion at the event horizon is

$$
\begin{aligned}
\Gamma & \propto \exp \left[-\operatorname{Im} \oint p_{r} d r\right] \\
& =\exp \left[-\operatorname{Im}\left(\int p_{r}^{\text {out }} d r-\int p_{r}^{\text {in }} d r\right)\right] \\
& =\exp \left[\mp 2 \operatorname{Im} \int p_{r}^{\text {out,in }} d r\right] .
\end{aligned}
$$

Here, $p_{r}=\partial_{r} W$, and out(in) correspond to $+(-)$. Thus the tunnelling rate is gotten as

$$
\Gamma \propto \exp \left[-2 \pi \frac{\omega-e A_{t+}}{f^{\prime}}(1+\beta \xi)\right] .
$$

However, the temporal contribution to the tunneling amplitude was missed in the above calculation [2, 5-7]. We use Kruskal coordinates $(T, R)$ to find this temporal contribution. The region exterior is described by

$$
T=e^{\kappa r_{*}} \sinh (\kappa t), \quad R=e^{\kappa r_{*}} \cosh (\kappa t),
$$


where $r_{*}=r+(1 / 2 \kappa) \ln \left(\left(r-r_{+}\right) / r_{+}\right)$is the tortoise coordinate and $\kappa$ is the surface gravity. The interior region is given by

$$
T=e^{\kappa r_{*}} \cosh (\kappa t), \quad R=e^{\kappa r_{*}} \sinh (\kappa t) .
$$

To find the temporal contribution, we connect these two patches across the horizon. Rotate the time $t$ as $t \rightarrow t-$ $(\pi / 2) i \kappa$. As pointed out in [2-4], this rotation would lead to an additional imaginary contribution coming from the temporal part, namely, $\operatorname{Im}\left[\left(\omega-e A_{t+}\right) \Delta t^{\text {out,in }}\right]=(1 / 2) \pi(\omega-$ $\left.e A_{t+}\right) \kappa$. So the total temporal contribution is $\operatorname{Im}[(\omega-$ $\left.\left.e A_{t+}\right) \Delta t\right]=\pi\left(\omega-e A_{t+}\right) \kappa$. Therefore, the tunnelling rate with the consideration of the temporal contribution is

$$
\begin{aligned}
\Gamma & \propto \exp \left[-\frac{1}{\hbar}\left(\operatorname{Im}\left(\left(\omega-e A_{t+}\right) \Delta t\right)+\operatorname{Im} \oint p_{r} d r\right)\right] \\
& =\exp \left[-4 \pi \frac{\omega-e A_{t+}}{f^{\prime}}\left(1+\frac{1}{2} \beta \xi\right)\right] .
\end{aligned}
$$

This is the Boltzmann factor with the Hawking temperature at the event horizon taking

$$
T=\frac{f^{\prime}}{4 \pi(1+\beta \xi)}=T_{0}\left(1-\frac{1}{2} \beta \xi\right),
$$

where $T_{0}=(1 / 2 \pi)\left(\alpha^{2} r_{+}+\left(2 M / \alpha r_{+}^{2}\right)-\left(4 Q^{2} / \alpha^{2} r_{+}^{3}\right)\right)$ is the standard Hawking temperature of the black string.

It is shown that the corrected temperature appears and is lower than the standard one. The correction is not only determined by the mass and charge of the black string, but also affected by the quantum number (mass, charge, and energy) of the emitted fermion. Quantum gravity correction slows down the increase of the Hawking temperature caused by the evaporation. Finally, the black string is in a balance state. At this state, the evaporation stops and the remnant is produced.

It is of interest to discuss the corrected area entropy. The entropy can be derived by the first law of thermodynamics with the corrected temperature (35). However, the expression is complicated, so we do not write it here. The corrected temperatures were also gotten in $[48-53]$. When $\beta=0$, the standard Hawking temperature is recovered [17, 47].

\section{Fermions' Tunnelling from a Rotating Black String}

In this section, we investigate uncharged fermions' tunnelling from the event horizon of a rotating black string. Therefore, effects of the electromagnetic field in the generalized Dirac equation are not taken into account here. The rotating black string solution in a spacetime asymptotically anti-de Sitter in the radial direction was derived by Lemos and Zanchin [54]. The solution is

$$
\begin{aligned}
d s^{2}= & -\left(\alpha^{2} r^{2}-\frac{4 M\left(1-a^{2} \alpha^{2} / 2\right)}{\alpha r}\right) d t^{2} \\
& +\left(\alpha^{2} r^{2}-\frac{4 M\left(1-(3 / 2) a^{2} \alpha^{2}\right)}{\alpha \mathrm{r}}\right)^{-1} d r^{2} \\
& -\frac{8 M a \sqrt{1-a^{2} \alpha^{2} / 2}}{\alpha r} d t d \varphi \\
& +\left(r^{2}-\frac{4 M a^{2}}{\alpha r}\right) d \varphi^{2}+\alpha^{2} r^{2} d z^{2},
\end{aligned}
$$

where $\alpha^{2}=-\Lambda / 3, \Lambda$ is the negative cosmological constant, and $a$ is the angular momentum per unit mass. It is defined that $a^{2} \alpha^{2}=1-\epsilon / M$ and $\epsilon=\sqrt{M^{2}-8 J^{2} \alpha^{2} / 9} . M$ and $J$ are the mass and angular momentum line densities of the spacetime, respectively. The relation between $J$ and $a$ is given by $J=$ $(3 / 2) M a \sqrt{1-a^{2} \alpha^{2} / 2}$. For convenience of the investigation, the metric (36) is rewritten as

$$
\begin{aligned}
d s^{2}= & -\Delta\left(\gamma d t-\frac{\delta}{\alpha^{2}} d \varphi\right)^{2}+r^{2}(\gamma d \varphi-\delta d t)^{2} \\
& +\frac{d r^{2}}{\Delta}+\alpha^{2} r^{2} d z^{2},
\end{aligned}
$$

where

$$
\begin{aligned}
& \Delta=\alpha^{2} r^{2}-\frac{b}{\alpha r}, \quad b=4 M\left(1-\frac{3 a^{2} \alpha^{2}}{2}\right), \\
& \gamma=\sqrt{\frac{2-a^{2} \alpha^{2}}{2-3 a^{2} \alpha^{2}}}, \quad \delta=\frac{a \alpha^{2}}{\sqrt{1-(3 / 2) a^{2} \alpha^{2}}} .
\end{aligned}
$$

The event horizon is located at $r_{+}=\alpha^{-1} b^{1 / 3}$ which is given for $\Delta=0$. To describe the fermion's tunnelling from the event horizon, one can directly construct a tetrad and gamma matrices from the metric (37). For simplicity, to construct the tetrad and gamma matrices, we perform the dragging coordinate transformation

$$
\varphi=\phi+\Omega t, \quad \Omega=\frac{-\Delta \gamma \delta \alpha^{2}+r^{2} \gamma \delta \alpha^{4}}{-\Delta \delta^{2}+r^{2} \gamma^{2} \alpha^{4}}
$$

on the metric (37) and get

$$
\begin{aligned}
d s^{2}= & -F(r) d t^{2}+\frac{1}{G(r)} d r^{2}+g_{\phi \phi} d \phi^{2}+g_{z z} d z^{2} \\
= & -\frac{\Delta r^{2}\left(\alpha^{2} \gamma^{2}-\delta^{2}\right)^{2}}{-\Delta \delta^{2}+\alpha^{4} r^{2} \gamma^{2}} d t^{2}+\frac{1}{\Delta} d r^{2} \\
& +\left(-\frac{\Delta \delta^{4}}{\alpha^{4}}+r^{2} \gamma^{2}\right) d \phi^{2}+\alpha^{2} r^{2} d z^{2} .
\end{aligned}
$$


Here we still only investigate the state with spin-up. Assume that the wave function of the fermion with spin-up state shares the same expression as (19), namely,

$$
\Psi=\left(\begin{array}{c}
A \\
0 \\
B \\
0
\end{array}\right) \exp \left(\frac{i}{\hbar} I(t, r, \phi, z)\right)
$$

The tetrad is easily constructed as

$$
e_{\mu}{ }^{a}=\operatorname{diag}\left(\sqrt{F}, 1 / \sqrt{G}, \sqrt{g_{\phi \phi}}, \sqrt{g_{z z}}\right)
$$

Now gamma matrices take on the form

$$
\begin{array}{ll}
\gamma^{t}=\frac{1}{\sqrt{F(r)}}\left(\begin{array}{cc}
i & 0 \\
0 & -i
\end{array}\right), & \gamma^{\phi}=\sqrt{g^{\phi \phi}}\left(\begin{array}{cc}
0 & \sigma^{1} \\
\sigma^{1} & 0
\end{array}\right), \\
\gamma^{r}=\sqrt{G(r)}\left(\begin{array}{cc}
0 & \sigma^{3} \\
\sigma^{3} & 0
\end{array}\right), & \gamma^{z}=\sqrt{g^{z z}}\left(\begin{array}{cc}
0 & \sigma^{2} \\
\sigma^{2} & 0
\end{array}\right) .
\end{array}
$$

In the above equations, $g^{\phi \phi}=\alpha^{4} /\left(-\Delta \delta^{4}+\alpha^{4} r^{2} \gamma^{2}\right), g^{z z}=$ $1 / \alpha^{2} r^{2}$. Inserting the wave function and the gamma matrices into the generalized Dirac equation and adopting the same process as the above section, we get

$$
\begin{aligned}
- & i A \frac{1}{\sqrt{F}} \partial_{t} I-B\left(1-\beta m^{2}\right) \sqrt{G} \partial_{r} I \\
- & A m \beta\left[g^{r r}\left(\partial_{r} I\right)^{2}+g^{\phi \phi}\left(\partial_{\phi} I\right)^{2}+g^{z z}\left(\partial_{z} I\right)^{2}\right] \\
+ & B \beta \sqrt{G} \partial_{r} I\left[g^{r r}\left(\partial_{r} I\right)^{2}+g^{\phi \phi}\left(\partial_{\phi} I\right)^{2}+g^{z z}\left(\partial_{z} I\right)^{2}\right] \\
+ & A m\left(1-\beta m^{2}\right)=0 \\
i B & \frac{1}{\sqrt{F}} \partial_{t} I-A\left(1-\beta m^{2}\right) \sqrt{G} \partial_{r} I \\
- & B m \beta\left[g^{r r}\left(\partial_{r} I\right)^{2}+g^{\phi \phi}\left(\partial_{\phi} I\right)^{2}+g^{z z}\left(\partial_{z} I\right)^{2}\right] \\
+ & A \beta \sqrt{G} \partial_{r} I\left[g^{r r}\left(\partial_{r} I\right)^{2}+g^{\phi \phi}\left(\partial_{\phi} I\right)^{2}+g^{z z}\left(\partial_{z} I\right)^{2}\right] \\
+ & B m\left(1-\beta m^{2}\right)=0, \\
A & \left\{-\left(1-\beta m^{2}\right) \sqrt{g^{\phi \phi}} \partial_{\phi} I+\beta \sqrt{g^{\phi \phi}} \partial_{\phi} I\right. \\
& \left.\times\left[g^{r r}\left(\partial_{r} I\right)^{2}+g^{\phi \phi}\left(\partial_{\phi} I\right)^{2}+g^{z z}\left(\partial_{z} I\right)^{2}\right]\right\}=0 \\
& \times\left[g^{r r}\left(\partial_{r} I\right)^{2}+g^{\phi \phi}\left(\partial_{\phi} I\right)^{2}+g^{z z}\left(\partial_{z} I\right)^{2}\right] \\
& i\left(1-\beta m^{2}\right) \sqrt{g^{z z}} \partial_{z} I+i \beta \sqrt{g^{z z}} \partial_{z} I
\end{aligned}
$$

$$
\begin{aligned}
B\{ & -\left(1-\beta m^{2}\right) \sqrt{g^{\phi \phi}} \partial_{\phi} I+\beta \sqrt{g^{\phi \phi}} \partial_{\phi} I \\
& \times\left[g^{r r}\left(\partial_{r} I\right)^{2}+g^{\phi \phi}\left(\partial_{\phi} I\right)^{2}+g^{z z}\left(\partial_{z} I\right)^{2}\right] \\
& -i\left(1-\beta m^{2}\right) \sqrt{g^{z z}} \partial_{z} I+i \beta \sqrt{g^{z z}} \partial_{z} I \\
& \left.\times\left[g^{r r}\left(\partial_{r} I\right)^{2}+g^{\phi \phi}\left(\partial_{\phi} I\right)^{2}\right]+g^{z z}\left(\partial_{z} I\right)^{2}\right\}=0 .
\end{aligned}
$$

It is also difficult to solve the action $I$ from the above equations. We first observe the last two equations. They can be reduced into the same equation and yield $\sqrt{g^{\phi \phi}} \partial_{\phi} I+$ $i \sqrt{g^{z z}} \partial_{z} I=0$. This implies

$$
g^{\phi \phi}\left(\partial_{\phi} I\right)^{2}+g^{z z}\left(\partial_{z} I\right)^{2}=0 .
$$

Now our interest is the first two equations which determine the Hawking temperature of the black string. Considering the properties of the metrics (36) and (40), we carry out separation of variables as

$$
I=-(\omega-j \Omega) t+W(r, z)+j \phi,
$$

where $\omega$ and $j$ are the energy and angular momentum of the emitted fermion, respectively. Inserting (47) into (44) and canceling $A$ and $B$ yield

$$
B_{6}\left(\partial_{r} W\right)^{6}+B_{4}\left(\partial_{r} W\right)^{4}+B_{2}\left(\partial_{r} W\right)^{2}+B_{0}=0
$$

where

$$
\begin{aligned}
& B_{6}=\beta^{2} G^{3} F, \\
& B_{4}=\beta G^{2} F\left(m^{2} \beta-2\right), \\
& B_{2}=G F\left[\left(1-\beta m^{2}\right)^{2}+2 \beta m^{2}\left(1-m^{2} \beta\right)\right], \\
& B_{0}=-m^{2}\left(1-\beta m^{2}\right)^{2} F-(\omega-j \Omega)^{2} .
\end{aligned}
$$

Neglecting the higher order terms of $\beta$ and solving (48) at the event horizon, we get the solution of $W$. Thus the imaginary part of $W$ is

$$
\begin{array}{rl}
I m W_{ \pm}= \pm \int d & d \sqrt{\frac{m^{2} F+(\omega-j \Omega)^{2}}{G F}} \\
& \times\left(1+\beta m^{2}+\beta \frac{(\omega-j \Omega)^{2}}{F}\right) \\
= & \pm \pi \frac{\left(\omega-j \Omega_{+}\right) \alpha^{4} r_{+}^{2} \gamma}{\left(2 \alpha^{4} r_{+}^{3}+b\right)\left(\alpha^{2} \gamma^{2}-\delta^{2}\right)}(1+\beta \chi),
\end{array}
$$

where $+(-)$ are the outgoing (ingoing) solutions, $\chi=$ $(3 / 2) m^{2}+3 j\left(\omega-j \Omega_{+}\right) \delta /\left(\alpha^{2} \gamma^{2}-\delta^{2}\right) \gamma r_{+}^{2}-(3 / 2)$ $\left(\left(\omega-j \Omega_{+}\right)^{2} \delta^{2} /\left(\alpha^{2} \gamma^{2}-\delta^{2}\right) r_{+}^{2}\right)$, and $\Omega_{+}=\delta / \gamma$ is the angular velocity at the event horizon. It is not difficult to prove that $\chi>0$. 
To find the temporal contribution, we use the Kruskal coordinates $(T, R)$. The region exterior to the string $\left(r>r_{+}\right)$ is described by

$$
T=e^{\kappa_{+} r_{*}} \sinh \left(\kappa_{+} t\right), \quad R=e^{\kappa_{+} r_{*}} \cosh \left(\kappa_{+} t\right),
$$

where $r_{*}=r+\left(1 / 2 \kappa_{+}\right) \ln \left(\left(r-r_{+}\right) / r_{+}\right)$and $\kappa_{+}$denote the surface gravity. The interior region is

$$
T=e^{\kappa_{+} r_{*}} \cosh \left(\kappa_{+} t\right), \quad R=e^{\kappa_{+} r_{*}} \sinh \left(\kappa_{+} t\right) .
$$

Adopting the same process as the above section, we get that the total temporal contribution is $\operatorname{Im}\left[\left(\omega-j \Omega_{+}\right) \Delta t\right]=\pi(\omega-$ $\left.j \Omega_{+}\right) \kappa_{+}$. Therefore, the tunnelling rate is

$$
\begin{aligned}
\Gamma & \propto \exp \left[-\frac{1}{\hbar}\left(\operatorname{Im}\left(\left(\omega-j \Omega_{+}\right) \Delta t\right)+\operatorname{Im} \oint p_{r} d r\right)\right] \\
& =\exp \left[-\frac{4 \pi\left(\omega-j \Omega_{+}\right) \alpha^{4} r_{+}^{2} \gamma}{\left(2 \alpha^{4} r_{+}^{3}+b\right)\left(\alpha^{2} \gamma^{2}-\delta^{2}\right)}\left(1+\frac{1}{2} \beta \chi\right)\right] .
\end{aligned}
$$

Equation (53) is the Boltzmann factor of the Hawking temperature at the event horizon taking

$$
T=\frac{\left(2 \alpha^{4} r_{+}^{3}+b\right)\left(\alpha^{2} \gamma^{2}-\delta^{2}\right)}{4 \pi \alpha^{4} r_{+}^{2} \gamma(1+(1 / 2) \beta \chi)}=T_{0}\left(1-\frac{1}{2} \beta \chi\right),
$$

where $T_{0}=\left(2 \alpha^{4} r_{+}^{3}+b\right)\left(\alpha^{2} \gamma^{2}-\delta^{2}\right) / 4 \pi \alpha^{4} r_{+}^{2} \gamma$ is the standard Hawking temperature. Obviously, the corrected Hawking temperature is lower than the standard one. The correction is related not only to the mass and angular momentum of the black string but also to the quantum number (mass, angular momentum, and energy) of the emitted fermion. Due to $\chi>$ 0 , there is a balance point. At this point, the evaporation stops and the remnant is left.

\section{Conclusion}

In this paper, taking into account the influence of quantum gravity, we modified the Dirac equation in curved spacetime by the modified fundamental commutation relations put forward in [33]. Then the tunnelling radiation of fermions from the event horizons of the charged and rotating black strings was investigated. The corrected Hawking temperatures were gotten. In the charged spacetime, the correction is related not only to the mass and charge of the black string but also to the quantum number (mass, charge, and energy) of the emitted fermion. In the rotating spacetime, the quantum number (mass, angular momentum, and energy) of the emitted fermion and the mass and angular momentum of the black string affect the Hawking temperature at the same time. Due to the quantum gravity corrections, the evaporation of the black strings slows down. Finally, the evaporation stops and the remnants are left. The remnants in the final state were also discussed in [55-58]. It is of interest to discuss remnants. A review of this topic can be found in [59].

When $\beta=0$, the standard Hawking temperatures are recovered.

\section{Conflict of Interests}

The authors declare that there is no conflict of interests regarding the publication of this paper.

\section{Acknowledgment}

This work is supported by the National Natural Science Foundation of China with Grant no. 11205125.

\section{References}

[1] P. Kraus and F. Wilczek, "Self-interaction correction to black hole radiance," Nuclear Physics B, vol. 433, no. 2, pp. 403-420, 1995.

[2] V. Akhmedova, T. Pilling, A. de Gill, and D. Singleton, "Temporal contribution to gravitational WKB-like calculations," Physics Letters B, vol. 666, no. 3, pp. 269-271, 2008.

[3] E. T. Akhmedov, T. Pilling, and D. Singleton, "Subtleties in the quasi-classical calculation of Hawking radiation," International Journal of Modern Physics D, vol. 17, no. 13-14, pp. 2453-2458, 2008.

[4] V. Akhmedova, T. Pilling, A. de Gill, and D. Singleton, "Comments on anomaly versus $\mathrm{WKB} /$ tunneling methods for calculating Unruh radiation," Physics Letters B, vol. 673, no. 3, pp. 227-231, 2009.

[5] E. T. Akhmedov, V. Akhmedova, T. Pilling, and D. Singleton, "Thermal radiation of various gravitational backgrounds," International Journal of Modern Physics A, vol. 22, no. 8-9, pp. 17051715, 2007.

[6] B. D. Chowdhury, "Problems with tunneling of thin shells from black holes," Pramana, vol. 70, no. 1, pp. 3-26, 2008.

[7] E. T. Akhmedov, V. Akhmedova, and D. Singleton, "Hawking temperature in the tunneling picture," Physics Letters B, vol. 642, no. 1-2, pp. 124-128, 2006.

[8] M. K. Parikh and F. Wilczek, "Hawking radiation as tunneling," Physical Review Letters, vol. 85, no. 24, pp. 5042-5045, 2000.

[9] K. Srinivasan and T. Padmanabhan, "Particle production and complex path analysis," Physical Review D, vol. 60, no. 2, Article ID 024007, 20 pages, 1999.

[10] S. Shankaranarayanan, T. Padmanabhan, and K. Srinivasan, "Hawking radiation in different coordinate settings: complex paths approach," Classical and Quantum Gravity, vol. 19, no. 10, pp. 2671-2687, 2002.

[11] M. Angheben, M. Nadalini, L. Vanzo, and S. Zerbini, "Hawking radiation as tunneling for extremal and rotating black holes," Journal of High Energy Physics, vol. 2005, no. 5, article 14, 2005.

[12] R. Kerner and R. B. Mann, "Tunnelling, temperature, and TaubNUT black holes," Physical Review D, vol. 73, no. 10, Article ID 104010, 11 pages, 2006.

[13] J. Zhang and Z. Zhao, "Hawking radiation of charged particles via tunneling from the Reissner-Nordström black hole," Journal of High Energy Physics, vol. 2005, no. 10, article 55, 2005.

[14] Q.-Q. Jiang and S.-Q. Wu, "Hawking radiation of charged particles as tunneling from Reissner-Nordström-de Sitter black holes with a global monopole," Physics Letters B, vol. 635, no. 2-3, pp. 151-155, 2006.

[15] R. Kerner and R. B. Mann, "Fermions tunnelling from black holes," Classical and Quantum Gravity, vol. 25, no. 9, Article ID 095014, 2008. 
[16] R. Kerner and R. B. Mann, "Charged fermions tunnelling from Kerr-Newman black holes," Physics Letters B, vol. 665, no. 4, pp. 277-283, 2008.

[17] J. Ahmed and K. Saifullah, "Hawking temperature of rotating charged black strings from tunneling," Journal of Cosmology and Astroparticle Physics, vol. 2011, no. 11, article 23, 2011.

[18] J. Ahmed and K. Saifullah, "Hawking radiation of Dirac particles from black strings," Journal of Cosmology and Astroparticle Physics, vol. 2011, no. 8, article 11, 2011.

[19] R. Li and J.-R. Ren, "Dirac particles tunneling from BTZ black hole," Physics Letters B, vol. 661, no. 5, pp. 370-372, 2008.

[20] D.-Y. Chen, Q.-Q. Jiang, and X.-T. Zu, "Hawking radiation of Dirac particles via tunnelling from rotating black holes in de Sitter spaces," Physics Letters B, vol. 665, no. 2-3, pp. 106-110, 2008.

[21] Q.-Q. Jiang, "Dirac particle tunneling from black rings," Physical Review D, vol. 78, no. 4, Article ID 044009, 8 pages, 2008.

[22] R. D. Criscienzo and L. Vanzo, "Fermion tunneling from dynamical horizons," Europhysics Letters, vol. 82, no. 6, Article ID 60001, 2008.

[23] S. A. Hayward, R. D. Criscienzo, M. Nadalini, L. Vanzo, and S. Zerbini, "Local hawking temperature for dynamical black holes," Classical and Quantum Gravity, vol. 26, no. 6, Article ID 062001, 2009.

[24] B. R. Majhi, "Fermion tunneling beyond semiclassical approximation," Physical Review D, vol. 79, no. 4, Article ID 044005, 6 pages, 2009.

[25] S. P. Robinson and F. Wilczek, "Relationship between Hawking radiation and gravitational anomalies," Physical Review Letters, vol. 95, no. 1, Article ID 011303, 4 pages, 2005.

[26] S. Iso, H. Umetsu, and F. Wilczek, "Hawking radiation from charged black holes via gauge and gravitational anomalies," Physical Review Letters, vol. 96, no. 15, Article ID 151302, 4 pages, 2006.

[27] R. Banerjee and B. R. Majhi, "Connecting anomaly and tunneling methods for the Hawking effect through chirality," Physical Review D, vol. 79, no. 6, Article ID 064024, 5 pages, 2009.

[28] P. K. Townsend, "Small-scale structure of spacetime as the origin of the gravitational constant," Physical Review D, vol. 15, no. 10, pp. 2795-2801, 1977.

[29] D. Amati, M. Ciafaloni, and G. Veneziano, "Can spacetime be probed below the string size?” Physics Letters B, vol. 216, no. 1-2, pp. 41-47, 1989.

[30] K. Konishi, G. Paffuti, and P. Provero, "Minimum physical length and the generalized uncertainty principle in string theory," Physics Letters B, vol. 234, no. 3, pp. 276-284, 1990.

[31] L. J. Garay, "Quantum gravity and minimum length," International Journal of Modern Physics A, vol. 10, no. 2, pp. 145-165, 1995.

[32] G. Amelino-Camelia, "Relativity in spacetimes with shortdistance structure governed by an observer-independent (Planckian) length scale," International Journal of Modern Physics D, vol. 11, no. 1, pp. 35-59, 2002.

[33] A. Kempf, G. Mangano, and R. B. Mann, "Hilbert space representation of the minimal length uncertainty relation," Physical Review D, vol. 52, no. 2, pp. 1108-1118, 1995.

[34] L. N. Chang, D. Minic, N. Okamura, and T. Takeuchi, "Effect of the minimal length uncertainty relation on the density of states and the cosmological constant problem," Physical Review D, vol. 65, no. 12, Article ID 125028, 7 pages, 2002.
[35] B. R. Majhi and E. C. Vagenas, "Modified dispersion relation, photon's velocity, and Unruh effect," Physics Letters B, vol. 725, no. 4-5, pp. 477-480, 2013.

[36] M. V. Battisti and G. Montani, "The big-bang singularity in the framework of a generalized uncertainty principle," Physics Letters B, vol. 656, no. 1-3, pp. 96-101, 2007.

[37] W. Chemissany, S. Das, A. F. Ali, and E. C. Vagenas, "Effect of the generalized uncertainty principle on post-inflation preheating," Journal of Cosmology and Astroparticle Physics, vol. 2011, no. 12, article 17, 2011.

[38] W. Kim, E. J. Son, and M. Yoon, "Thermodynamics of a black hole based on a generalized uncertainty principle," Journal of High Energy Physics, vol. 2008, no. 1, article 35, 2008.

[39] L. Xiang and X. Q. Wen, "A heuristic analysis of black hole thermodynamics with generalized uncertainty principle," Journal of High Energy Physics, vol. 2009, no. 10, article 46, 2009.

[40] A. Bina, S. Jalalzadeh, and A. Moslehi, "Quantum black hole in the generalized uncertainty principle framework," Physical Review D, vol. 81, no. 2, Article ID 023528, 7 pages, 2010.

[41] K. Nozari and S. Saghafi, "Natural cutoffs and quantum tunneling from black hole horizon," Journal of High Energy Physics, vol. 2012, no. 11, article 5, 2012.

[42] D. Chen, H. Wu, and H. Yang, "Fermion's tunnelling with effects of quantum gravity," Advances in High Energy Physics, vol. 2013, Article ID 432412, 6 pages, 2013.

[43] W. Greiner, Relativistic Quantum Mechanics: Wave Equation, Springer, Berlin, Germany, 2000.

[44] K. Nozari and M. Karami, "Minimal length and generalized Dirac equation," Modern Physics Letters A, vol. 20, no. 40, pp. 3095-3103, 2005.

[45] S. Hossenfelder, M. Bleicher, S. Hofmann, J. Ruppert, S. Scherer, and H. Stöcker, "Signatures in the Planck regime," Physics Letters $B$, vol. 575, no. 1-2, pp. 85-99, 2003.

[46] J. P. S. Lemos, “Three-dimensional black holes and cylindrical general relativity," Physics Letters B, vol. 353, no. 1, pp. 46-51, 1995.

[47] R.-G. Cai and Y.-Z. Zhang, "Black plane solutions in fourdimensional spacetimes," Physical Review D, vol. 54, no. 8, pp. 4891-4898, 1996.

[48] R. Banerjee and B. R. Majhi, "Quantum tunneling and back reaction," Physics Letters B, vol. 662, no. 1, pp. 62-65, 2008.

[49] R. Banerjee, B. R. Majhi, and S. Samanta, "Noncommutative black hole thermodynamics," Physical Review D, vol. 77, no. 12, Article ID 124035, 8 pages, 2008.

[50] R. Banerjee and B. R. Majhi, "Quantum tunneling beyond semiclassical approximation," Journal of High Energy Physics, vol. 2008, no. 6, article 95, 2008.

[51] R. Banerjee and B. R. Majhi, "Quantum tunneling and trace anomaly," Physics Letters B, vol. 674, no. 3, pp. 218-222, 2009.

[52] B. R. Majhi and S. Samanta, "Hawking radiation due to photon and gravitino tunneling," Annals of Physics, vol. 325, no. 11, pp. 2410-2424, 2010.

[53] R. Banerjee, C. Kiefer, and B. R. Majhi, "Quantum gravitational correction to the Hawking temperature from the LemaitreTolman-Bondi model," Physical Review D, vol. 82, no. 4, Article ID 044013, 7 pages, 2010.

[54] J. P. S. Lemos and V. T. Zanchin, "Rotating charged black strings and three-dimensional black holes," Physical Review D, vol. 54, no. 6, pp. 3840-3853, 1996. 
[55] R. J. Adler, P. Chen, and D. I. Santiago, "The generalized uncertainty principle and black hole remnants," General Relativity and Gravitation, vol. 33, no. 12, pp. 2101-2108, 2001.

[56] F. Scardigli, C. Gruber, and P. Chen, "Black hole remnants in the early universe," Physical Review D, vol. 83, no. 6, Article ID 063507, 30 pages, 2011.

[57] R. Banerjee and S. Ghosh, "Generalised uncertainty principle, remnant mass and singularity problem in black hole thermodynamics," Physics Letters B, vol. 688, no. 2-3, pp. 224-229, 2010.

[58] L. Xiang, "A note on the black hole remnant," Physics Letters B, vol. 647 , no. 2-3, pp. 207-210, 2007.

[59] P. Nicolini, "Noncommutative black holes, the final appeal to quantum gravity: a review," International Journal of Modern Physics A, vol. 24, no. 7, pp. 1229-1308, 2009. 

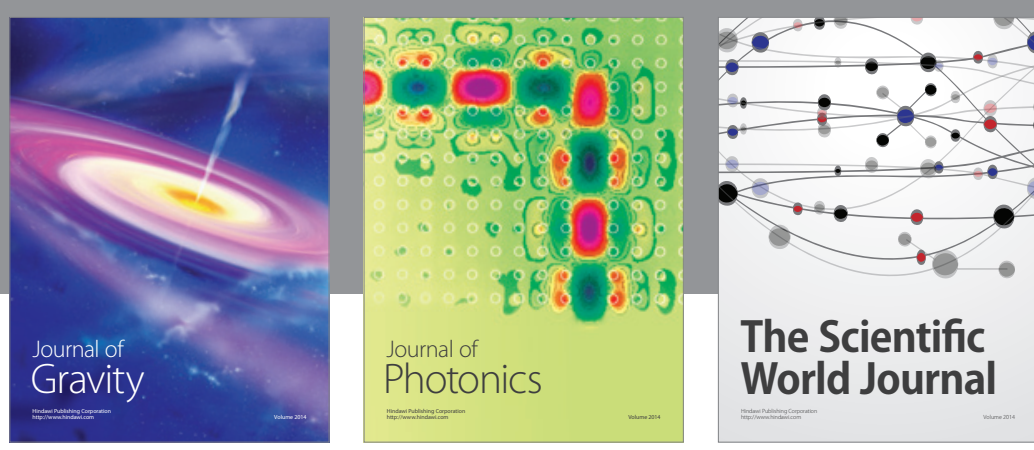

The Scientific World Journal
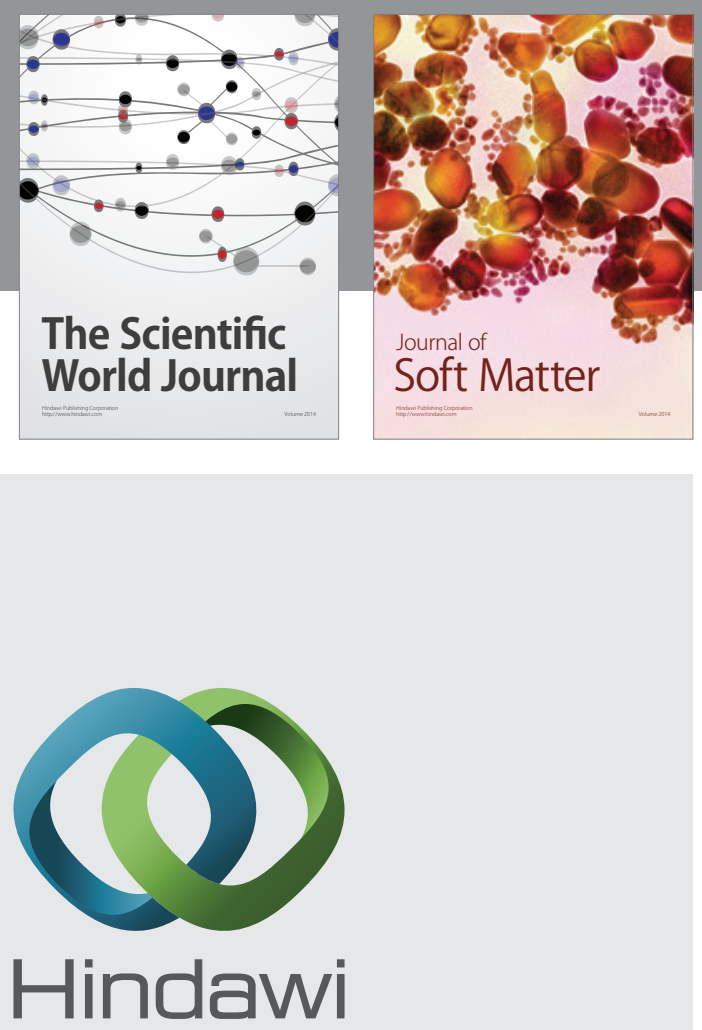

Submit your manuscripts at

http://www.hindawi.com

nternational Journal of

Statistical Mechanics
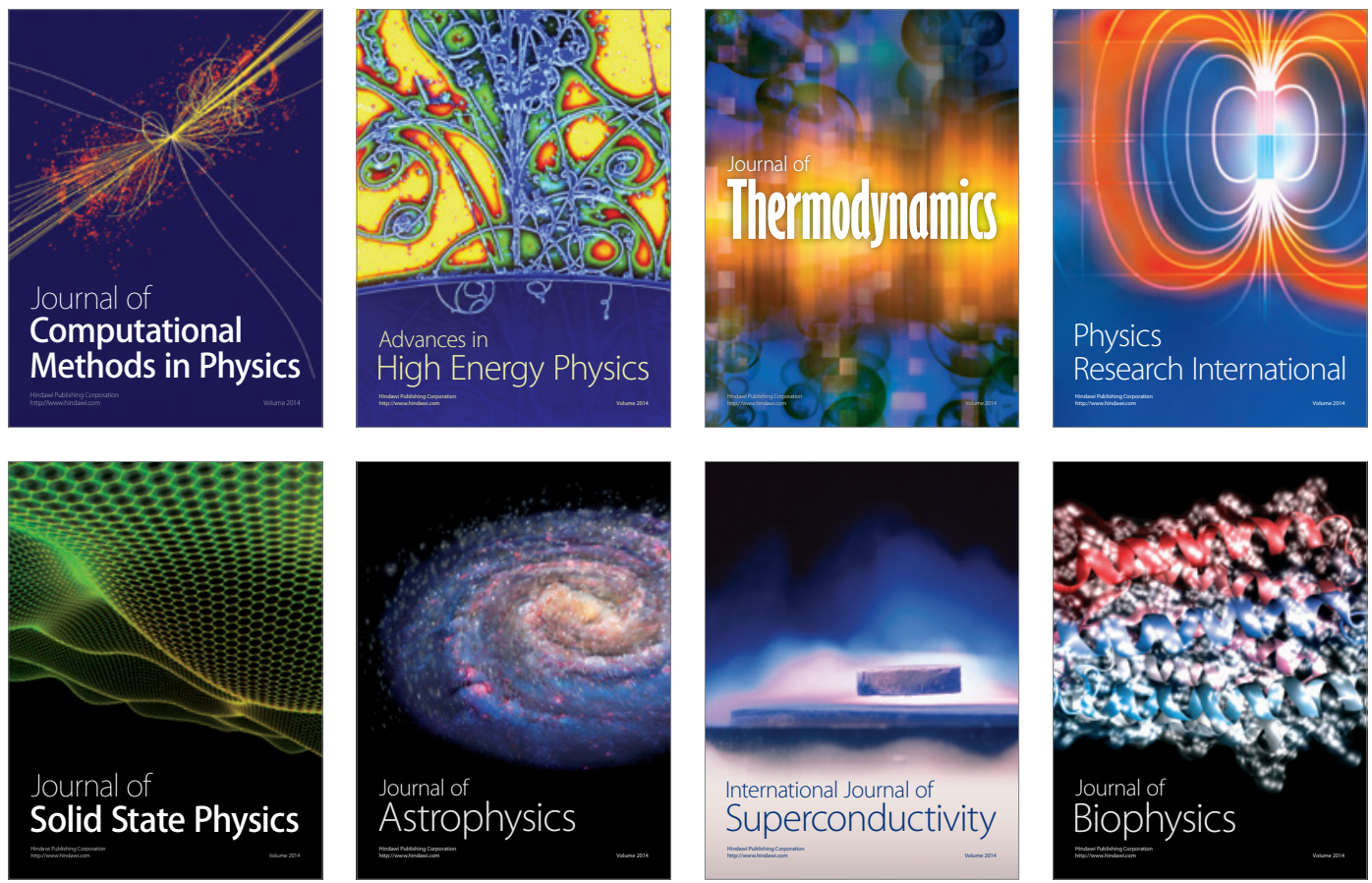
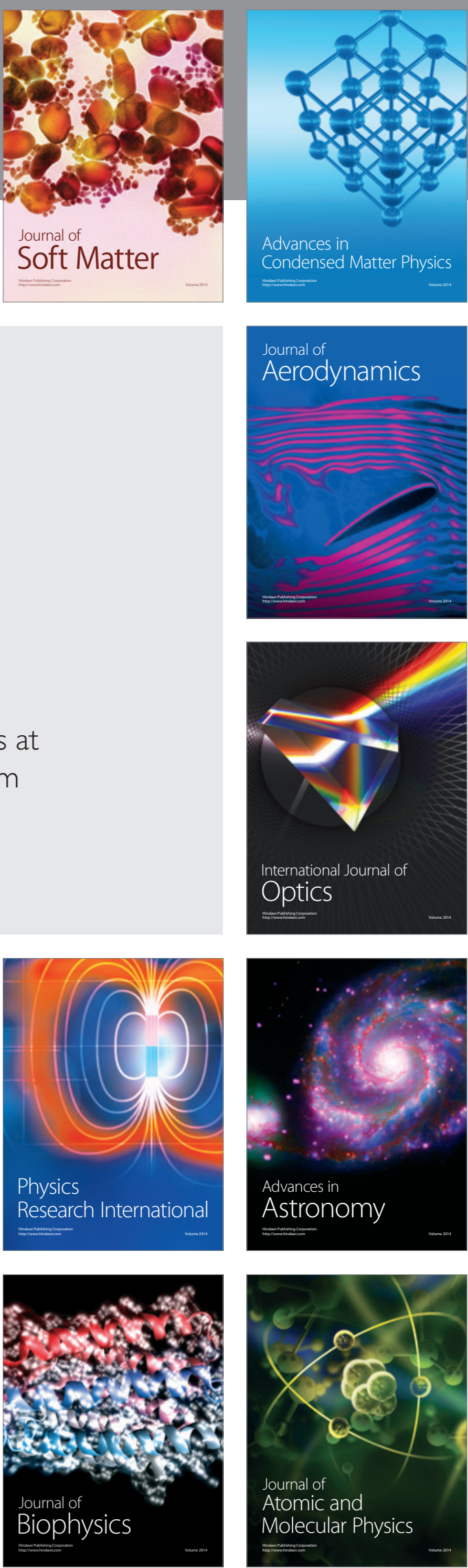